\title{
Robotic Walkers for Children and Youth with Cerebral Palsy: A Review of Past Successes and Ongoing Advancement
}

\author{
Anna McCormick ${ }^{1,2}$, Hana Alazem ${ }^{1,2}$, Carolyn Hunt ${ }^{3}$, Sarah Zaidi', Corrie Dixon ${ }^{3}$ \\ ${ }^{1}$ Children's Hospital of Eastern Ontario \\ 401 Smyth Road, Ottawa, Canada \\ amccormick@cheo.on.ca; halazem@cheo.on.ca; szaidi@cheo.on.ca \\ ${ }^{2}$ University of Ottawa \\ 451 Smyth Road, Ottawa, Canada \\ ${ }^{3}$ Grandview Children's Centre \\ 600 Townline Rd S, Oshawa, ON L1H 7K6 \\ dr.carolyn.hunt@grandviewkids.ca; corrie.dixon@grandviewkids.ca
}

\begin{abstract}
Each child with a disability deserves the support required to achieve their maximum physical potential, optimal participation and an active lifestyle throughout their lifespan. In this paper, we aim to highlight past successes, present work and share a vision for future advancements in robotic gait technologies that enable walking in children and youth with severe physical disabilities. We will summarize work which demonstrates how a robotic walker with a lift and body weight support system has shown great promise in mobilization, exercise and participation in youth and young adults with Cerebral Palsy (CP). This study was conducted in 3 phases; 1) a case study, 2) a pilot study evaluating mobility in a clinical setting, and 3) a study focusing on training and participation in a community setting. Secondly, we will describe the planned assessment of the Trexo Plus, a walker and wearable lower extremity robotic device combination, in younger children with significant physical involvement secondary to CP. This device allows precise repetition of normal gait patterns while controlling range of motion at the hip, knee and ankle. Lastly, we will highlight international innovative robotic work which has produced a novel robotic platform with a partial body weight support system (PBWS), active system for adapting hip height and an exoskeleton with joint motion control. A multimodal human-robot interface (MHRI) accompanies the unique walker system which assesses intent to move, posture and balance and gait patterns. This system has the potential to allow therapists to construct and precisely evaluate unique robot facilitated programming. There is a new generation of robotic devices providing the means for individuals with $\mathrm{CP}$ to walk independently. With a look to the future, we endeavour to evaluate and facilitate the development of this robotic technology as it is integrated into clinical care, activity enhancement and community participation.
\end{abstract}

Keywords: Robotics, Power walker, Cerebral palsy, Technology, Mobility.

\section{Mobile Robotic Walker with Integrated Lift: The SoloWalk \\ 1.1. Introduction}

In 2016, our Ontario based research team was excited to witness and report the case of a 16-year-old youth with mixed tone quadriplegia cerebral palsy (CP) walking with the aid of the SoloWalk robotic walker [1]. This youth was a level IV on the Gross Motor Function Classification System (GMFCS) and had not preformed this activity in over 2 years. Typically, individuals with GMFCS IV CP have limited self-mobility, use assistive devices to move within their environment and require assistance during transfers [2]. The SoloWalk, a robotic walking device with built in power lift, was developed to assist adults in early mobilization in acute or rehabilitation settings [3]. This walker became the first of its kind to be adapted for youth with cerebral palsy and tested in a clinical setting.

\subsection{Device: The SoloWalk Robotic Walker}

Investigators from the biorobotic research laboratory at Carleton University's Advanced Biomechatronics and Locomotion laboratory (presently Gaitronics Inc.), in consultation with adult medical and rehabilitation communities, created the SoloWalk robotic walker. This robotic device was initially designed to focus on early mobilization for adults. [2]. Figure 1 shows the SoloWalk powered mobility training device used for our first case study that enabled our participant with cerebral palsy to mobilize [4]. 


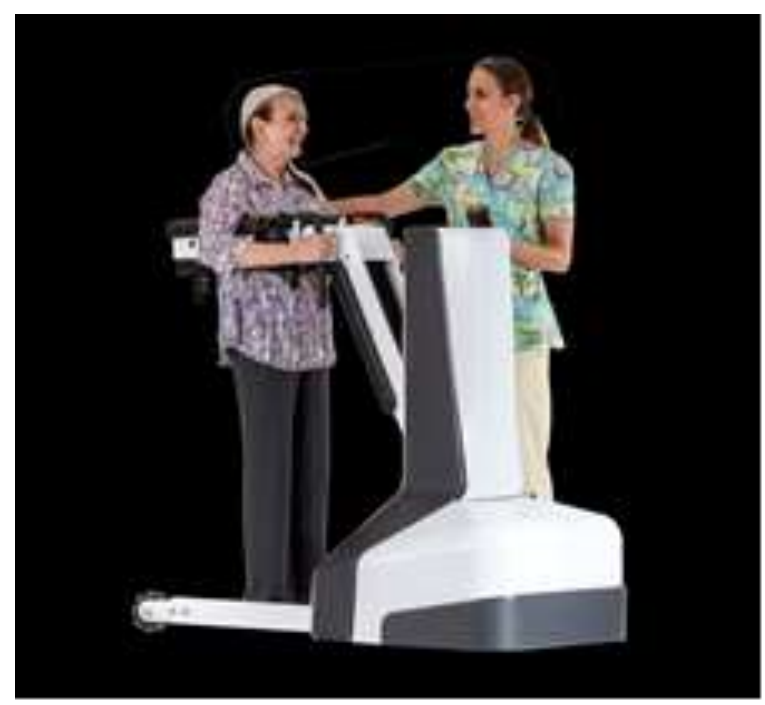

Fig. 1: SoloWalk Device.

The SoloWalk's key features include servo-powered omnidirectional wheels on the mobile base of the device, and a body-weight support (BWS) system used for lifting patients, and preventing falls [3]. The SoloWalk also has a reactive control system based on robotic force control techniques. A force sensor mounted between the frame of the device and the harness measures force inputs from the patient. The proprietary control system uses this information to command SoloWalk to move in the direction chosen by the user. This control system guarantees stable patient-device interactions and automatically synchronizes the device's motion with the users.

Therapists can use a wired remote control to activate the key functions of the device, such as speed and direction. With this remote, therapists can allow a patient to initiate steps on their own (i.e., device automatically follows user's voluntary motion), or directly control the motion of the device in any direction to guide their motion as required to achieve therapeutic goals.

SoloWalk is also equipped with numerous safety features [3]:

i. Safety switches may be controlled by the Health Care Professional (HCP) and or patient. These switches allow the device to lock into place during an emergency. This is an attractive feature when using the device with children and adolescents with $\mathrm{CP}$ who can fatigue quickly [5].

ii. A harness system prevents a patient from falling. This system comprises of a torso and mating pelvis harness, which attaches between a patient's legs. The pelvis harness provides support from below and relieves pressure on an individual's shoulders when a user is suspended in the device.

iii. Software safety features for limiting the speed of the device and/or the direction of motion of the device. The HCP can customize the speed and motion limits according to each patient's need for support.

iv. The integrated lift and body weight support system: HCP injuries can be avoided since caregivers are no longer required to intervene and physically support a patient, or stabilize the patient if the patient loses their balance. The device monitors key data such as the patient's walking speed, pelvis height, and the amount of support required to remain upright. In the event that the patient suddenly loses their balance, it quickly and automatically locks into place to support the patient and prevent a fall.

\subsection{Objectives}

The SoloWalk device was studied in three phases and each phase had its own objectives. During the initial case study, our aim was to demonstrate the effective use of the SoloWalk by an individual with spastic quadriplegia secondary to cerebral palsy GMFCS IV [1]. Study objectives included identifying the advantages and disadvantages of a powered walker prototype and providing recommendations for modifications to assist in the development of a powered walker tailored for individuals with CP [1]. Following this case study (Phase 1), we conducted a pilot study with a small sample size to further explore the utility and any need for further device modifications (Phase 2) [6]. Finally, we moved the modified device into the community 
and looked at its effectiveness in exercise training, activity promotion and participation using an upgraded prototype (Phase 3) $[7,8]$.

\subsection{Results}

The successful case study was reported in the Proceedings of the 3rd International Conference of Control, Dynamic Systems, and Robotics 2016 [1]. A 16 year old male with spastic quadriplegia GMFCS IV walked within the Children's Treatment Centre for 40 meters and no complications were reported.

The pilot study for the second phase was a qualitative case series that identified the benefits and challenges of device use in youth and young adults with CP. We included five CP patients between the ages of 13 to 22 with GMFCS level IV enrolled at the Children's Hospital of Eastern Ontario [6]. Four of the five clients were able to walk at least 40 meters with the device. The one client unable to utilize the device had severe generalized dystonia (sustained abnormal postures). All responses to open ended questions were audio recorded and transcribed. Frequently appearing themes were documented in a Mind Map illustration (Figure 2).

Feedback from our participants and families was quite positive. Focus group recordings indicated that participants' felt safe and comfortable in the harness. Clients stated that it was "fairly easy" to get into and they liked the way the robot "lifted [them] up". Most felt well supported in the device, their arms and legs were comfortable and the movement of the robot was interpreted as smooth. The walker was responsive, it made it easier for them to walk and easy to turn. Individuals were very happy to walk, described the device as "cool" and stated that they would like to use it again. Family feedback included a written letter to the team stating "I have read articles over the years since my grandson was born and dreaming of the day that this might occur. Your group is to be commended!" - Participant's Grandfather [6].

The therapists were hopeful that the device could be made smaller, the harness could be better customized for comfort and support, and that the device could be adapted for uneven terrain/ different environments (i.e. carpet and sports fields). Overall, it was not difficult for the therapists to get the patient into the device and the technique of preparing the patient was not perceived as difficult to learn. It was in the opinion of the therapists that efficiency would be gained over time; however therapist control of the device was preferred rather than complete control by the user [6]. In Figure 2 we see the physical and social advantages and disadvantages of the powered walker identified during the case study trial [6].

There were recommended design changes to the robotic device. In general, participants' were quite pleased but some recommended more padding on the front and in the groin area of the harness. One client noted that the robot was "big, but he could get used to it." The clinicians noted that a child that may be passive in their approach appeared motivated to move in this device. They were surprised that one participant, with such decreased physical and cognitive involvement, could walk using this device for approximately 40 meters during his 60-minute assessment. He could use it with the assistance of the therapists and independently both facing the tower, holding on to the handles and in reverse in a hands free position [6].

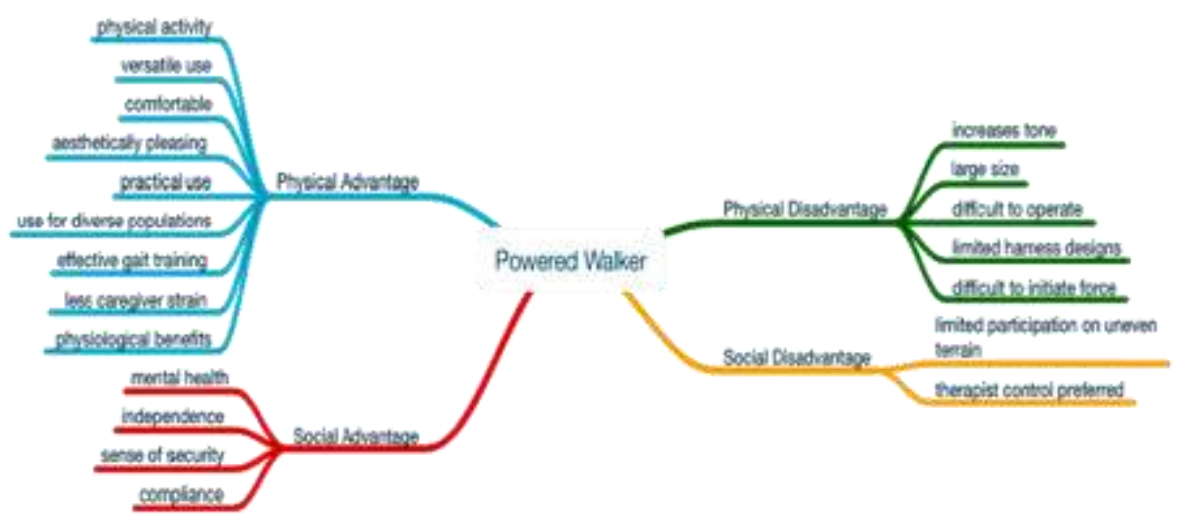

Fig. 2: Mind Map illustration of feedback given under major sub-themes. 
Modifications were made to the harness to increase comfort and overall support. As trials continue, we aspire to adapt the SoloWalk to improve function on uneven terrains (e.g. baseball diamond or sports played on the ice) and in smaller settings for use in homes.

Phase 3 of this pilot is ongoing, and we have added a collaborative testing site, the Whitby Abilities Centre in Ontario, Canada. We are continuing to collect information regarding the device design and assessment of participation in fitness activities such as running, throwing and kicking. Early data has demonstrated successful completion of these activities. There has been documentation of heart rate values moving into a range expected with moderate to intense exercise and client enjoyment while participating in a standing position. To date, no further recommendations have been made for changes to the robotic device by the research team, participants and/or caregivers [7,8]. Figure 3 and 4 shows the current SoloWalk device that is used for this phase of our study [9].

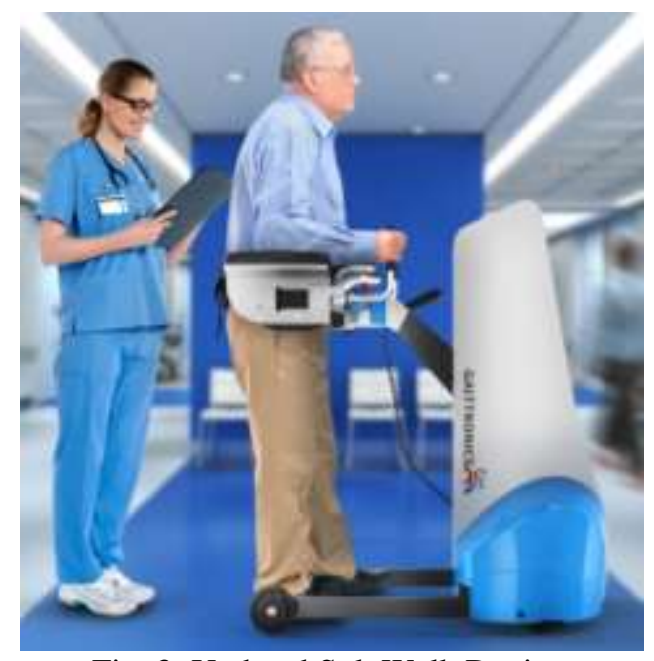

Fig. 3: Updated SoloWalk Device.

As we continue to test this device, we are exploring a fourth phase of investigation. Testing in phase 4 will include youth with $\mathrm{CP}$ transitioning to adult care as well as expanded study populations; including patients with acquired brain injury and stroke in both inpatient and outpatient settings. As resources shrink within hospital settings, there is a growing concern regarding staff injuries and limited time for exercise. Devices that lift and allow independent walking without risk of falls or health care practitioner injury offer viable options that need further testing. The intention of this collaborative work between therapists, physicians and engineers, is to create a device that will benefit a large number of clients with severe mobility challenges in multiple settings.

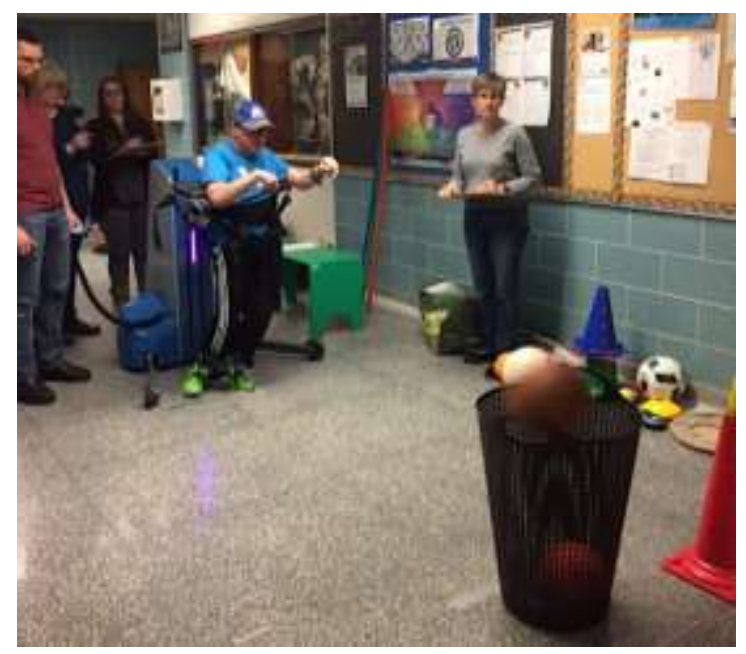

Fig. 4: Adolescent with CP demonstrates potential for functional activity and participation. 


\section{Trexo Plus: Walker and Wearable Lower Extremity Robotic Device Combination 2.1. Introduction}

There is now a walker and wearable lower extremity robotic device combination available in pediatric sizes fitting children as young as 2 years of age. In-depth research regarding the impact on developing children with neurodevelopmental disabilities is lacking.

\subsection{Device: The Trexo Plus}

The Trexo Plus system is a Health Canada approved robotic gait assistance device created by Trexo Robotics. This device is one of the first of its kind in North America, combining a pediatric mobile robotic walker with wearable robotic technology that can assist and support individuals as they mobilize independently. The Trexo Plus is adjustable in height and width to accommodate a wide range of ages starting as young as two years. It has been designed for clinical rehabilitation, permitting physiotherapists with the ability to deliver gait therapy using a robotic system. The creators describe potential use in children with conditions including, but not limited to, cerebral palsy, spina bifida, various genetic conditions, traumatic and non-traumatic brain injury and spinal cord injury [10].

The Trexo Plus system is controlled via a tablet that can modify gait speed, joint angles, and level of support. It provides power to lower extremity regions of articulation (hips and knees) through wearable robotic technology. The goal is to optimize supportive gait training and maintain range of motion. This combination of technology offers facilitated, repetitive precision movement. There is augmentation of power that is generated by the client and the capability to independently move and explore surrounding environments. The mobile nature of this device opens new opportunities for development, participation and inclusion while maintaining good form and limiting risk of fall or injury [10].

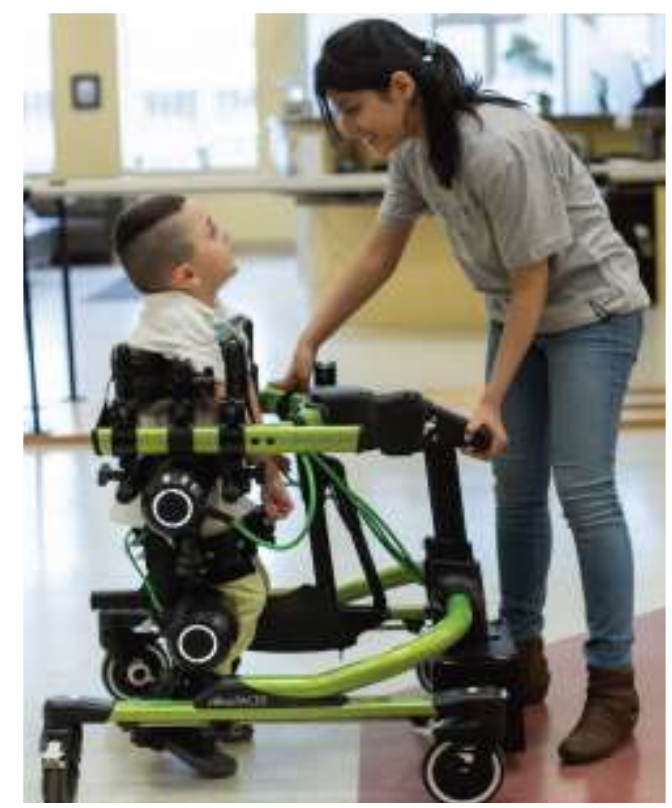

Fig. 4: Trexo plus Device.

\subsection{Objective}

Presently, our research team at the Children's Hospital of Eastern Ontario is developing a protocol to examine the impact of this newly available form of robotic technology in children with cerebral palsy. Our pilot study will determine the benefits and challenges of training with this robotic device compared to routine clinical care, for children ages 3 to 6 years who primarily use walkers or wheelchairs. We wish to study its influence on physical function, development, participation, and social integration. We aim to collaborate with a number of international centers that are also preparing to test this device.

Our primary objective is to evaluate a newly designed pediatric robotic device in therapy, more specifically gait training in children with cerebral palsy. We will aim to answer questions such as: Does the use of this cutting edge technology facilitate measureable changes including walking speed, endurance and gait quality over time? Are these changes greater 
than in therapeutic interventions without the device? Does the technology facilitate individualized goal attainment? Does it enable client safety, security, a positive therapy experience and participation in healthy activity? Are there improvements in tone, decreased contracture formation and improved bone health?

\subsection{Results}

There is potential for this technology to not only change the way therapy is delivered but also change the way children, with moderate to severe physical disabilities, function and participate. With increased use and development of wearable robotic technology, there is a potential to incorporate walking as an integral part of therapy and daily life in children who have limited or no ability to walk independently.

Primary outcome measures will assess a child's ability to walk using their manual device, the distance walked and speed of walking. Secondary objectives will examine a more global impact of this technology. Data will be collected to examine impact on quality of gait, child or proxy chosen individualized goals, feedback regarding safety, security, therapy experience and facilitation of participation. Impacts on tone and bone health will also be examined. We predict a positive impact and envision robotic technology transforming delivery of therapy and enhancing activity and participation in children with significant physical limitations.

\section{New and Exciting International Developments \\ 3.1. Introduction}

We foresee new and innovative robotic technologies on the horizon. Technology is evolving from simple walkers and exoskeletons to robotics-based tele rehabilitation, socially assistive robots and voice or brain activated robotic devices. The use of technology with increasing complexity has been on the rise with objectives well beyond reducing physical stress on health care practitioners. With the use of novel therapy strategies and increasing the design modularity on robotic devices, gait trainers and exoskeletons - robotic-based therapies can be customized to patient's needs. Exercises can be individualized, activity encouraged and patient engagement maximized [11]. We have chosen one particularly novel robotic walker, the CPWalker to highlight multiple new alternatives for gait training in individuals with neuromotor conditions.

\subsection{Device: CPWalker}

The CPWalker is a smart walker exoskeleton combination, developed by the Robotics and Automation Arganda Laboratory in del Rey, Spain. Bayón et al (10) describe a unique walking device that combines a novel robotic platform with a drive system, a partial body weight support system (PBWS), an active system for adapting hip height and an exoskeleton with joint motion control. There is also child-device interaction via a multimodal human-robotic interface. Electroencephalographic (EEG) is utilized to study intent to move, inertial measurement units provide feedback regarding posture and a laser range finder provides information regarding limb movement.

The CPWalker is described as having a number of training modes that encourage different forms of participation [12]. Position control mode applies a pre-set gait pattern to the lower limbs so the patient can perform and learn a walking motion sequence. Impedance control modes allow deviations from pre-set gait patterns. This setting promotes increased active participation within set limits. Finally, in zero-force control mode, the patient is in full control with minimal resistance from the device. The patient is creating the gait pattern while the device provides stability.

The multiple facets of this device add a new level of advancement in the field of robotics. The device allows free movement within the child's environment, individualized exercise programs with variable levels of patient control, and robotic augmentation. Additionally, there is central nervous system engagement and monitoring with regular feedback to encourage improvements. The potential for this device is promising and we await further research to highlight the impact of this technology in the realms of body function, activity and participation.

\subsection{Conclusion}

Technology advancement in the field of robotics, when developed collaboratively with clients and family at the center, has great potential. Early evidence supporting robotic-based therapies gives hope for improvements in the health and wellbeing for populations with significant motor limitations. Prevention of secondary complications (e.g. severe contracture) may decrease morbidity and hospitalization rates, benefiting the individuals in question and an overburdened health care system [5]. A close relationship amongst patients, families, clinicians, engineers, technologists and private industry is required for advancement. 
One can envision robotic devices allowing more intensive intervention in shorter time periods and enabling groupbased therapy, home programs and treatment in remote locations. With current therapist shortages and long waitlists of deserving clients, these efficiencies could greatly benefit families and clinical institutions. Options in the field of robotics, such as the SoloWalk, Trexo Plus and CPWalker to name a few, may put children and adults with severe disabilities on their feet, exploring the environment around them and participating in activities they love. Our ultimate goal is to find the right device, for the right person, at the right time.

\section{References}

[1] A. McCormick, H. Alazem, A. Morbi, R. Beranek, R. Adler, G. Tibi, E. Vilé "Power Walker Helps a Child with Cerebral Palsy," in Proceedings of the $3^{\text {rd }}$ International Conference of Control, Dynamic Systems, and Robotics, Ottawa, CA, 2016.

[2] P. Rosenbaum, R. Palisano, S. Walter, D. Russell, E. Wood, B. Galuppi, "Development and reliability of a system to classify gross motor function in children with cerebral palsy," Dev. Med. Child Neurol., vol. 39, no. 4, pp. 214-23, 1997.

[3] A. Morbi, "Design, control, and implementation of a robotic gait rehabilitation system for over ground gait training," Ph.D. dissertation, Dept. Mechanical and Aerospace Engineering, Carleton University, Ottawa, ON, 2013.

[4] GaitTronics Technology (2012). GaitTronics: Safe Early Mobilization for Patients [Online]. Available: http://gaittronics.com/

[5] J. Rimmer, J. Rowland, K. Yamaki. "Obesity and secondary conditions in adolescents with disabilities: addressing the needs of an underserved population," J. Adolesc. Health, vol. 41, no. 3, pp. 224-9, 2007.

[6] H. Alazem, A. McCormick, S. G. Nicholls, G. Tibi, E. Vilé, R. Adler, "Development of a powered mobility assistance device for individuals with cerebral palsy," Arch. Phys. Med. Rehabil., vol. 97, no. 10, pp. 85, 2016.

[7] McCormick et al., "Robotic Walker Tested for Exercise and Participation in Youth with Cerebral Palsy GMFCS IV," Dev. Med. Child Neuro., vol. 60, pp. 68, 2018.

[8] McCormick et al., "Development of a Powered Mobility Assistance Device for Adolescents with Cerebral Palsy," in Proceedings of the $31^{\text {st }}$ European Academy of Childhood Disability, Paris, FR, 2019. Presentation in preparation.

[9] Gaitronics. (2018). SoloWalk [Online]. Available: https://www.designdirectory.com/federaldesignhouse/SoloWalk

[10] Trexo Robotics, User's Manual (Small device) Trexo plus Version 0.9 - joint trajectory control with velocity. Mississauga, ON: Trexo Robotics Inc, 2018.

[11] Bayon et al., "Development and evaluation of a novel robotic platform for gait rehabilitation in patients with Cerebral Palsy: CPWalker," Rob. Auton. Syst., vol. 91, pp. 101-114, 2017.

[12] Bayon et al., "A robot-based gait training therapy for pediatric population with cerebral palsy: goal setting, proposal and preliminary clinical implementation, " J. Neuroeng. Rehabil., vol. 15, pp. 69, 2018. 